\title{
PROPER REGULAR SEMIGROUPS
}

\author{
F. E. MASAT ${ }^{1}$
}

\begin{abstract}
In a recent paper, D. B. McAlister gave several characterizations of proper inverse semigroups. In this paper, the concept of proper is extended to the class of regular semigroups. This is done by requiring that the set of idempotents of the semigroup coincides with the kernel of the minimum group congruence on the semigroup. A theorem is presented which contains several characterizations of proper regular semigroups, and the related result of McAlister then follows as a corollary. The paper concludes with some open questions and examples.
\end{abstract}

1. Introduction. Proper inverse semigroups were first developed by T. Saito in [9]. In [7], D. B. McAlister gave several characterizations of proper inverse semigroups. In this paper, the concept of properness is extended to the class of regular semigroups, and the resulting consequences are described.

After $\S 2$, which gives some preliminaries and definitions, $\$ 3$ presents the main theorem which contains several characterizations of proper regular semigroups. The related result of McAlister then follows as a corollary. The paper concludes with some examples and open questions.

2. Preliminaries. The notation of Clifford and Preston ([1], [2]) will be used throughout the paper. If $S$ is regular and there is no danger of ambiguity, $E$ will be used to denote $E_{S}$, the set of idempotents of $S$. The set of inverses of an element $c \in S$ will be denoted by $V(c)$.

When a congruence $\rho$ is such that $S / \rho$ is the maximal homomorphic image of $S$ of type $C$, as in [2, p. 275] and [2, Theorem 11.25 (A), p. 276], then $\rho$ will be called the minimum congruence on $S$ of type $C$ and $S / \rho$ will be called the maximum homomorphic image of $S$ of type $C$. In other words, $S / \rho$ is the maximum $C$-image if and only if $\rho$ is of type $C$ and $\rho \subseteq \sigma$ for each congruence $\sigma$ which is of type $C$.

If $\sigma$ is a group congruence on $S$, then $H$, the group identify of $S / \sigma$, satisfies the following properties due to R. Stoll [8, Theorem 3, p. 477]:

(i) $H$ is a subsemigroup of $S$;

(ii) if $a, b \in S$ and $a b \in H$ then $b a \in H$;

Presented to the Society, April 25, 1977; received by the editors July 19, 1977.

AMS (MOS) subject classifications (1970). Primary 20M10, $20 \mathrm{M} 15$.

Key words and phrases. Proper, group congruence, reflexiveness, inverse semigroups, unitary semigroups, orthodox semigroups.

${ }^{1}$ This research was supported by a Glassboro State College Faculty Research Grant. 
(iii) if $a, b \in S$ and $a$ and $a b \in H$ then $b \in H$;

(iv) $H$ is a neat subset of $S$.

Conversely, if $H$ is a subset of $S$ satisfying (i)-(iv), then there exists a group congruence $\sigma$, given by

$$
\sigma=\{(a, b) \in S \times S: x a, x b \in H \text { for some } x \in S\}
$$

such that $H$ is the group identity of $S / \sigma$.

Recall from [2, p. 17] that a subset $H$ is called neat if for each $s \in S$, there exist an $x \in S$ and $y \in S$ such that $s x \in H$ and $y s \in H$. Clifford and Preston [2, pp. 55, 56] call a subset $H$ of $S$ reflexive if it satisfies (ii) of (2.1) and left unitary if it satisfies (iii) of (2.1). Since the kernel of a congruence on a regular semigroup is the union of all the congruence classes containing an idempotent, the subsemigroup $H$, above, must necessarily contain $E$. In the following, the kernel of the minimum group congruence will be denoted by $K$.

Proper inverse semigroups were developed and investigated by McAlister in [7]. From [7, p. 352], an inverse semigroup is proper if and only if for $a \in S$ and $e \in E$, ea $=e$ implies that $a \in E$. We extend this notion of proper to regular semigroups by calling a regular semigroup $S$ proper if for $a \in S$ and $e \in E$, ea $\in E$ implies that $a \in E$. By the following lemma, we see that this extension, when restricted to inverse semigroups, reduces to the former definition.

LEMMA 2.2 Let $S$ be an inverse semigroup with set of idempotents $E$. If $e, e a \in E$ for some $a \in S$, then there exists an $f \in E$ such that $f=f a=a f$.

Proof. By [5, Lemma 2.2, p. 149] $e a=g \in E \Rightarrow a e=h \in E$. Letting $f=g h=e g h$ we have: $f a=e g h a=h g e a=h g=f$ and $a f=a e g h=a e h g=$ $h g=f$.

Lastly, the following result from T. E. Hall [3] is also needed.

LEMMA 2.3 [3, Theorem 3, p. 200]. If $S$ is an orthodox semigroup, then the relation $\delta$ defined by

$$
\delta=\{(a, b) \in S \times S: V(a)=V(b)\}
$$

is the minimum inverse semigroup congruence on $S$.

3. A characterization theorem. In this section a theorem is given which generalizes Proposition 1.1 of McAlister [7]. A lemma is needed before proceeding to the theorem.

LEMMA 3.1 Let $S$ be a regular semigroup with set of idempotents $E$.

(1) $S$ is orthodox if and only if for $a, b \in S, V(a) \cap V(b) \neq \varnothing$ implies that $V(a)=V(b)$.

(2) If $V\left(a^{2}\right)=V(a)$ for some $a \in S$, then $a \in E$.

Proof. Part (1) is [4, Theorem VI. 1.10, p. 189]. For (2), suppose that $V\left(a^{2}\right)=V(a)$ for some $a \in S$. Since $a^{\prime} \in V(a)$ implies that $a^{\prime} \in V\left(a^{2}\right)$, then $a^{\prime} a^{2} a^{\prime}=a^{\prime}$. Thus $a^{2}=\left(a a^{\prime} a\right)\left(a a^{\prime} a\right)=a\left(a^{\prime} a^{2} a^{\prime}\right) a=a a^{\prime} a=a$. 
THEOREM 3.2 Let $S$ be a regular semigroup. For $S$, denote the set of idempotents by $E$, the minimum group kernel by $K$, the minimum inverse congruence on $S$ by $\delta$, and the minimum group congruence on $S$ by $\sigma$. The following are equivalent.

(1) $S$ is proper.

(2) $S$ is orthodox and $\sigma \cap R \subseteq \delta$.

(3) $S$ is orthodox and $S / \delta$ is proper.

(4) $E$ is a left unitary subset of $S$.

(5) $E=K$.

(6) $S$ is right unitary.

Proof. Parts (1) and (4) are equivalent since the definition of proper implies that $E$ is left unitary. Numbers (4) and (5) are equivalent by Theorem 3.3 of [6]. From [5, Lemma 2.1, p. 149], (4) and (6) are equivalent.

We next show that (2) and (5) are equivalent. Let $E=K$ and suppose $a, b \in S$ such that $(a, b) \in \sigma \cap \Re$. Since $E=K$, then $a \sigma b$ implies that $a b^{\prime}, a^{\prime} b \in E$. Thus $b^{\prime} a \Re b^{\prime} b$ and $a^{\prime} a \Re a^{\prime} b$ imply that $b^{\prime} a b^{\prime} b=b^{\prime} b$ and $a^{\prime} b a^{\prime} a$ $=a^{\prime} a$. It follows then that

$$
b^{\prime} a b^{\prime}=b^{\prime} \text { and } a^{\prime} b a^{\prime}=a^{\prime} .
$$

Also, $a \Re b \Re a a^{\prime} \Re b b^{\prime}$ and hence $a a^{\prime} b=b$ and $b b^{\prime} a=a$. Combining this with equation (3.3) yields $b b^{\prime} a b^{\prime} a=b b^{\prime} a$ and $a a^{\prime} b a^{\prime} b=a a^{\prime} b$; i.e.,

$$
a b^{\prime} a=a \text { and } b a^{\prime} b=b .
$$

Since $E=K, S$ is orthodox and therefore equations (3.3) and (3.4) and Lemma 3.1 imply that $V(a)=V(b)$. By Lemma $2.3,(a, b) \in \delta$.

Conversely, suppose that $\sigma \cap \Re \subseteq \delta$ and let $e, e a \in E$ for some $a \in S$.

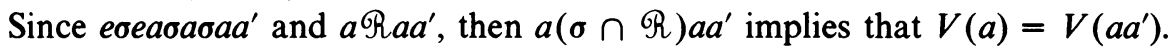
Thus $a a^{\prime} \in V(a)$ and therefore $a\left(a a^{\prime}\right) a=a$; i.e., $a^{2}=a$ and $a \in E$.

Lastly, we show that (1) and (3) are equivalent. Suppose that $S$ is proper and let $x \in E_{S / \delta}, y \in S / \delta$ such that $x=x y$. By [4, Lemma II.4.6, p. 52] there exists $e \in E$ such that $e \delta=x$. Letting $b \in S$ such that $b \delta=y$, then $e b \delta=x y=x=e \delta$ implies that $V(e b)=V(e)$. Thus $e b(e) e b=e b$ and therefore $e b \in E$. Since $S$ is proper, then $b \in E$ and it follows that $y \in E_{S / \delta}$; i.e., $S / \delta$ is proper.

Conversely, if $e \in E, a \in S$ such that $e a \in E$, it follows that $e \delta,(e a) \delta \in$ $E_{S / \delta}$ and therefore that $a \delta \in E_{S / \delta}$. This implies that $a^{2} \delta=a \delta$; i.e., that $V\left(a^{2}\right)=V(a)$, and therefore by Lemma 3.1, $a \in E$ and $S$ is proper.

Corollary 3.5 [7, Proposition 1.1, p. 352]. Let $S$ be an inverse semigroup with semilattice of idempotents $E$ and maximum group homomorphic image $G=S / \sigma$. Then the following are equivalent.

(1) $S$ is proper.

(2) $\sigma \cap \Re=\Delta$ where $\Delta$ is the diagonal of $S \times S$.

(3) $\sigma^{\natural}$ is one-to-one on each $\Re$-class of $S$.

(4) The map $\phi: S \rightarrow E \times G$ defined by $s \phi=\left(s s^{-1}\right.$, so $\left.\sigma^{\natural}\right)$ is one-to-one.

(5) $e=e^{2}=$ ae implies $a^{2}=a$. 
Proof. Since $S$ is inverse, then in Theorem 3.2, $\delta=\Delta$ and (1)-(4) are equivalent as in [7, Proposition 1.1, p. 152]. By Lemma 2.2, (1) and (5) are equivalent.

COROLlARY 3.6 If $S$ is a regular semigroup and $E$ is a rectangular band, then $E=R=K$.

Proof. Since $S$ is the product of a group and a rectangular band, $K=E$ follows from Corollary 3.5. Note that Corollary 3.6 extends [6, Corollary 4.5, p. 401] where it was shown that $E=R$.

For inverse semigroups, part (2) of Corollary 3.5 could be rephrased as: $S$ is proper if and only if $\sigma \cap \mathcal{H}=\Delta$. In the regular case we have the following:

Proposition 3.7 Let $S$ be a regular semigroup. Denote the minimum group congruence on $S$ by $\sigma$ and the identity relation on $S$ by $\Delta$.

(1) If $\sigma \cap R=\Delta$, then $S$ is proper.

(2) If $S$ is proper, then $\sigma \cap \mathcal{H}=\Delta$.

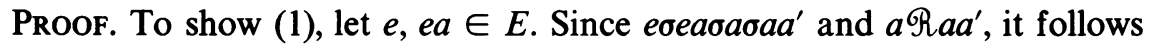
that $a=a a^{\prime}$ and thus $a^{2}=a$.

For (2), the proof of part (2) of Theorem 3.2 yields that $b^{\prime} a b^{\prime}=b^{\prime}$, $a=\left(b b^{\prime}\right) a$, and $\left(b^{\prime} a\right) b^{\prime} b=b^{\prime} a$. Dually for $\mathcal{L}, a\left(b^{\prime} b\right)=a$ and $b^{\prime} a\left(b^{\prime} b\right)=b^{\prime} a$. Thus $b^{\prime} b=b^{\prime} a$ and we have $a=a\left(b^{\prime} b\right)=\left(b b^{\prime}\right) a\left(b^{\prime} b\right)=b\left(b^{\prime} a b^{\prime}\right) b=b b^{\prime} b$ $=b$.

Note that the converses of Proposition 3.7 are open questions. For examples of proper regular semigroups in which $E, R$, and $K$ are described, see Exercises IV.4 (p. 125), and V.10 and V.11 (p. 182) in [4]. In these particular examples, $E=R \subseteq K$ and conditions are given in order that $E=K$.

ACKNOWLEDGEMENTS. The author wishes to express his gratitude to the referee for his many valuable comments and suggestions. In particular, the examples at the end of the paper were suggested by the referee in lieu of a section which essentially developed the same results.

\section{REFERENCES}

1. A. H. Clifford and G. B. Preston, The algebraic theory of semigroups. I, Math. Surveys, no. 7, Amer. Math. Soc., Providence, R. I., 1961; reprinted 1964.

2. __ The algebraic theory of semigroups. II, Math. Surveys, no. 7, Amer. Math. Soc., Providence, R. I., 1967; reprinted with corrections, 1971.

3. T. E. Hall, On regular semigroups whose idempotents form a subsemigroup, Bull. Austral. Math. Soc. 6 (1969), 195-208.

4. J. M. Howie, An introduction to semigroup theory, London Math. Soc., London, 1976.

5. J. M. Howie and G. Lallement, Certain fundamental congruences on a regular semigroup, Proc. Glasgow Math. Assoc. 7 (1966), 145-159.

6. F. E. Masat, Right group and group congruences on a regular semigroup, Duke Math. J. 40 (1973), 393-402.

7. D. B. McAlister, Groups, semilattices and inverse semigroups. II, Trans. Amer. Math. Soc. 196 (1974), 351-370.

8. R. R. Stoll, Homomorphisms of a semigroup onto a group, Amer. J. Math. 73 (1951), 475-481.

9. T. Saito, Proper ordered inverse semigroups, Pacific J. Math. 15 (1965), 649-666.

Department of Mathematics, Glassboro State College, Glassboro, New Jersey 08028 